\title{
Polarization in HIC: comparison of methods
}

\author{
Georgy Prokhorov ${ }^{1, *}$, Oleg Teryaev ${ }^{1,2,3, * *}$, and Valentin Zakharov ${ }^{2,4, * * *}$ \\ ${ }^{1}$ Joint Institute for Nuclear Research, 141980 Dubna, Russia \\ ${ }^{2}$ Institute of Theoretical and Experimental Physics, B. Cheremushkinskaya 25, 117218 Moscow, Russia \\ ${ }^{3}$ National Research Nuclear University MEPhI (Moscow Engineering Physics Institute), Kashirskoe \\ Shosse 31, 115409 Moscow, Russia \\ ${ }^{4}$ School of Biomedicine, Far Eastern Federal University, 690950 Vladivostok, Russia
}

\begin{abstract}
Based on the Wigner function for an medium with thermal vorticity, an exact non-perturbative formula for axial current was obtained. It is confirmed that the Chiral Vortical Effect results from the Wigner function. It is shown that the angular velocity and acceleration play the role of new chemical potentials, which is expressed in the appearance of combination $\mu \pm(\Omega \pm i|\boldsymbol{a}|) / 2$. It is shown that acceleration enters in the form of imaginary chemical potential and the consequences of this fact are investigated. An expression for the boundary temperature for a medium of fermions, which simultaneously has acceleration and rotation, is derived. This temperature in the particular case coincides with the temperature of Unruh.
\end{abstract}

\section{Introduction}

Recently, a variety of remarkable effects have been discovered, which lie on the border of relativistic hydrodynamics and quantum field theory. Two famous effects of this kind are Chiral Magnetic (CME) and Chiral Vortical (CVE) effects [1-8]. These effects lead to macroscopic transport phenomena in the plasma of elementary particles, which should be observed in experiment. Thus, it was shown that the rotation of a liquid of massless fermions - a chiral fluid - leads to the appearance of an axial current along an angular velocity, which is the essence of the CVE. It is remarkable that, at the same time, the nature of these effects turns out to be closely connected with the various most fundamental properties of matter, in particular, quantum anomalies [3-5,9]. In particular, it was shown that CVE is a direct consequence of the electromagnetic axial anomaly [3-5].

The standard formula for the CVE contains only linear terms in 4-velocity derivatives, the occurrence of which is dictated by the electromagnetic anomaly (more accurately, this corresponds to the linear terms with chemical potential). At the same time, higher-order terms seem to be associated with other anomalies of quantum field theory, in particular, gravitational [9]. Therefore, the analysis of these terms is to allow a better understanding of the effect of other quantum anomalies on transport phenomena. This is one of the objectives of this work.

On the other hand, it is important to understand the role of acceleration in transport phenomena. So, in $[10,11]$ it was shown that in a medium with acceleration there is a lower

\footnotetext{
*e-mail: prokhorov@ theor.jinr.ru

**e-mail: teryaev@theor.jinr.ru

***e-mail: vzakharov@itep.ru
} 
bound on the temperature. Analysis of the role of acceleration and a deeper analysis of the role of rotation in transport phenomena is another task of this paper.

There are various approaches to the study of chiral effects. We will use a quantumstatistical approach based on the covariant Wigner function for a medium with thermal vorticity, proposed in [12]. This Wigner function was used in analysing the effects of non-stationary motion of a medium in the energy-momentum tensor, vector and axial current, and other observable quantities $[6,12,13]$. In particular, the calculation of the polarization of baryons in heavy ion collisions is based on it [14].

Note that there are other approaches to the calculation of polarization, in particular, based on the calculation of the anomalous axial charge of strange quarks [15]. Different approaches predict the same behavior of the polarization of baryons in heavy ion collisions. We are discussing the sources of such compliance.

In the first step, we derive a formula for the axial current in the general case of massive fermions [16]. For this purpose we summarize the full series in thermal vorticity, and therefore the expression obtained is non-perturbative. We show that the resulting formula leads to CVE in the linear approximation, confirming the result of [6]. On the next step, the role of the angular velocity and acceleration is analysed and it is shown that they play the role of new chemical potentials, which leads to the appearance of the combination $\mu \pm(\Omega \pm i|\boldsymbol{a}|) / 2$ (look [17]). From the fact that the angular velocity plays the role of a chemical potential, it follows that the axial current is suppressed in region $\Omega<2(m-|\mu|)$ at $a_{\mu}=0, T=0$. At the same time, the acceleration appears as an imaginary chemical potential, which leads to instability in the axial current below the Unruh temperature $T<T_{U}$.

\section{Derivation of an exact non-perturbative formula for axial current based on the Wigner covariant function}

A mathematical object containing information on acceleration, rotation, and temperature gradient in the medium is the thermal vorticity tensor

$$
\varpi_{\mu \nu}=-\frac{1}{2}\left(\partial_{\mu} \beta_{v}-\partial_{v} \beta_{\mu}\right)
$$

In a state of global non-stationary thermodynamic equilibrium, the next relations are satisfied

$$
\beta_{\mu}=b_{\mu}+\varpi_{\mu \nu} x_{v}, \quad b_{\mu}=\text { const }, \quad \varpi_{\mu \nu}=\text { const }, \quad \xi=\frac{\mu}{T}=\text { const } .
$$

The Wigner function, taking into account the effects associated with thermal vorticity, proposed in [12], is expressed in terms of the distribution function $X(x, p)$, which has the form of a modified Fermi-Dirac distribution

$$
X(x, p)=\left(\exp \left[\beta_{\mu} p^{\mu}-\xi\right] \exp \left[-\frac{1}{2} \varpi_{\mu \nu} \Sigma^{\mu v}\right]+I\right)^{-1}
$$

where $\Sigma_{\mu \nu}=\frac{i}{4}\left[\gamma_{\mu}, \gamma_{v}\right]$. Note that (3) is an ansatz. However, this formula leads to the correct limiting cases [12], and also satisfies the zeroth-order kinetic equation with the vanishing collision term [18].

The average value of the axial current in a non-stationary medium with thermal vorticity can be calculated using (3) by averaging over the momentum space

$$
\left\langle j_{\mu}^{5}\right\rangle=-\frac{1}{16 \pi^{3}} \epsilon_{\mu \alpha \nu \beta} \int \frac{d^{3} p}{\varepsilon} p^{\alpha}\left\{\operatorname{tr}\left(X \Sigma^{\nu \beta}\right)-\operatorname{tr}\left(\bar{X} \Sigma^{\nu \beta}\right)\right\} .
$$

In $[16,17]$, an exact formula was obtained for the axial current from (4) by expanding (3) into a Taylor series in $\varpi$, calculation of a trace in each term of the series, and summing the 
resulting series back. In this sense, the resulting formula can be considered an exact nonperturbative result. As a result, the following formula was obtained for global equilibrium

$$
\begin{aligned}
\left\langle j_{\mu}^{5}\right\rangle= & \frac{\omega_{\mu}+i \operatorname{sgn}(\omega a) a_{\mu}}{2\left(g_{\omega}-i g_{a}\right)} \int \frac{d^{3} p}{(2 \pi)^{3}}\left\{n_{F}\left(E_{p}-\mu-g_{\omega} / 2+i g_{a} / 2\right)-n_{F}\left(E_{p}-\mu+g_{\omega} / 2\right.\right. \\
& \left.\left.-i g_{a} / 2\right)+n_{F}\left(E_{p}+\mu-g_{\omega} / 2+i g_{a} / 2\right)-n_{F}\left(E_{p}+\mu+g_{\omega} / 2-i g_{a} / 2\right)\right\}+c . c .
\end{aligned}
$$

where $n_{F}(E)=\left(e^{E / T}+1\right)^{-1}$ is the Fermi-Dirac distribution, $a^{\mu}$ is acceleration $a^{\mu}=u^{v} \partial_{v} u^{\mu}$ and $\omega_{\mu}$ is vorticity $\omega_{\mu}=\frac{1}{2} \epsilon_{\mu \nu \alpha \beta} u^{\nu} \partial^{\alpha} u^{\beta}$ and scalar combinations $g_{a}$ and $g_{\omega}$ have the form

$$
\begin{aligned}
& g_{\omega}=\frac{1}{\sqrt{2}}\left(\sqrt{\left(a^{2}-\omega^{2}\right)^{2}+4(\omega a)^{2}}+a^{2}-\omega^{2}\right)^{1 / 2}, \\
& g_{a}=\frac{1}{\sqrt{2}}\left(\sqrt{\left(a^{2}-\omega^{2}\right)^{2}+4(\omega a)^{2}}-a^{2}+\omega^{2}\right)^{1 / 2} .
\end{aligned}
$$

It is useful to consider a particular case by going into the comoving reference system, in which $a^{\mu}=(0, \boldsymbol{a})$ and $\omega^{\mu}=(0, \boldsymbol{\Omega})$, and assuming that $\boldsymbol{\Omega} \| \boldsymbol{a}$, that is, the acceleration directed along the rotation axis. Then (5) gives

$$
\begin{array}{r}
\left\langle\boldsymbol{j}^{5}\right\rangle=\frac{1}{2} \int \frac{d^{3} p}{(2 \pi)^{3}}\left\{n_{F}\left(E_{p}-\mu-\frac{\Omega}{2}+i \frac{|\boldsymbol{a}|}{2}\right)-n_{F}\left(E_{p}-\mu+\frac{\Omega}{2}+i \frac{|\boldsymbol{a}|}{2}\right)+\right. \\
\left.n_{F}\left(E_{p}+\mu-\frac{\Omega}{2}+i \frac{|\boldsymbol{a}|}{2}\right)-n_{F}\left(E_{p}+\mu+\frac{\Omega}{2}+i \frac{|\boldsymbol{a}|}{2}\right)+c . c .\right\} \boldsymbol{e}_{\Omega} .
\end{array}
$$

It can be seen from (7) that the angular velocity and acceleration appear in combination with the chemical potential

$$
\mu \rightarrow \mu \pm(\Omega \pm i|\boldsymbol{a}|) / 2 .
$$

Thus, we can say that the angular velocity plays the role of a real chemical potential, while acceleration plays the role of an imaginary chemical potential. The following sections will explore the consequences of this curious fact.

\section{Massless limit}

In the limit $m \rightarrow 0$ (5) gives

$$
\begin{array}{r}
\left\langle j_{\mu}^{5}\right\rangle=\left(\frac{1}{6}\left[T^{2}+\frac{a^{2}-\omega^{2}}{4 \pi^{2}}\right]+\frac{\mu^{2}}{2 \pi^{2}}\right) \omega_{\mu}+\frac{1}{12 \pi^{2}}(\omega a) a_{\mu}+\omega_{\mu}\left[-\frac{4 \pi T g_{a}}{g_{a}^{2}+g_{\omega}^{2}}\left(\frac{T^{2}}{6}+\frac{\mu^{2}}{2 \pi^{2}}-\right.\right. \\
\left.\left.\frac{g_{a}^{2}}{8 \pi^{2}}-\frac{g_{\omega}^{2}}{8 \pi^{2}}\right)\left\lfloor\frac{g_{a}}{4 \pi T}+\frac{1}{2}\right\rfloor-2 T^{2}\left\lfloor\frac{g_{a}}{4 \pi T}+\frac{1}{2}\right\rfloor^{2}+\frac{8 \pi T^{3} g_{a}}{3\left(g_{a}^{2}+g_{\omega}^{2}\right)}\left\lfloor\frac{g_{a}}{4 \pi T}+\frac{1}{2}\right\rfloor^{3}\right]+ \\
a_{\mu} \operatorname{sgn}(\omega a)\left[-\frac{4 \pi T g_{\omega}}{g_{a}^{2}+g_{\omega}^{2}}\left(\frac{T^{2}}{6}+\frac{\mu^{2}}{2 \pi^{2}}+\frac{g_{a}^{2}}{8 \pi^{2}}+\frac{g_{\omega}^{2}}{8 \pi^{2}}\right)\left\lfloor\frac{g_{a}}{4 \pi T}+\frac{1}{2}\right\rfloor+\frac{8 \pi T^{3} g_{\omega}}{3\left(g_{a}^{2}+g_{\omega}^{2}\right)}\left\lfloor\frac{g_{a}}{4 \pi T}+\frac{1}{2}\right\rfloor^{3}\right],
\end{array}
$$

where $\lfloor\cdot\rfloor$ is the integer part. For $T>\widetilde{T}_{U}$, where $\widetilde{T}_{U}$ is

$$
\widetilde{T}_{U}=\frac{g_{a}}{2 \pi},
$$

formula (9) gives

$$
\left\langle j_{\mu}^{5}\right\rangle=\left(\frac{1}{6}\left[T^{2}+\frac{a^{2}-\omega^{2}}{4 \pi^{2}}\right]+\frac{\mu^{2}}{2 \pi^{2}}\right) \omega_{\mu}+\frac{1}{12 \pi^{2}}(\omega a) a_{\mu} .
$$


In the linear approximation in $\partial_{\mu} u_{v}$, we have standard formula for CVE [1-8]

$$
\left\langle j_{\mu}^{5}\right\rangle=\left(\frac{T^{2}}{6}+\frac{\mu^{2}}{2 \pi^{2}}\right) \omega_{\mu} .
$$

Thus, CVE is a direct consequence of the Wigner function. Note that the term $\frac{\omega^{2}}{24 \pi^{2}} \omega_{\mu}$ was obtained previously [1,9], in particular, its relationship with the gravitational anomaly was shown [9].

\section{Angular velocity as chemical potential}

Let's consider the limit $a_{\mu}=0, T=0$. Then the formula (7) can be exactly integrated and expressed in the terms of Heaviside function

$$
\begin{aligned}
\left\langle j^{5}\right\rangle= & \frac{1}{6 \pi^{2}}\left\{\theta\left(\mu+\frac{\Omega}{2}-m\right)\left[\left(\mu+\frac{\Omega}{2}\right)^{2}-m^{2}\right]^{3 / 2}-\theta\left(\mu-\frac{\Omega}{2}-m\right)\left[\left(\mu-\frac{\Omega}{2}\right)^{2}-m^{2}\right]^{3 / 2}+\right. \\
& \left.\theta\left(-\mu+\frac{\Omega}{2}-m\right)\left[\left(\mu-\frac{\Omega}{2}\right)^{2}-m^{2}\right]^{3 / 2}-\theta\left(-\mu-\frac{\Omega}{2}-m\right)\left[\left(\mu+\frac{\Omega}{2}\right)^{2}-m^{2}\right]^{3 / 2}\right\} \boldsymbol{e}_{\Omega} .
\end{aligned}
$$

It is known that in a stationary environment at $T=0$ different physical quantities are zero for $|\mu|<m$. Taking into account the replacement (8), we can expect similar effects for the angular velocity. Indeed, analysing (13), we see that the axial current is zero in a two-dimensional region $\Omega<2(m-|\mu|)$ in the coordinates $\Omega$ and $\mu$. The corresponding graph is shown on Fig. 1, on the left hand side.
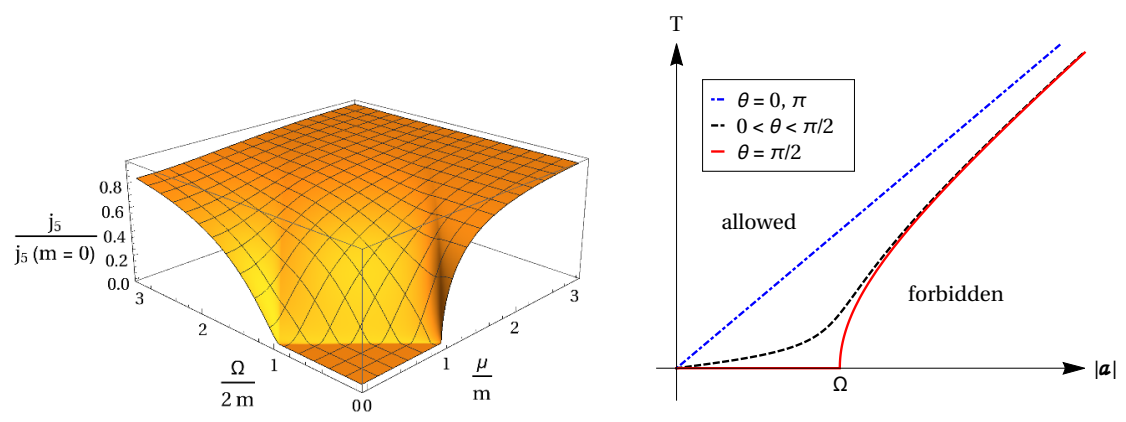

Figure 1. Left: axial current (13), as a function of the chemical potential and angular velocity at zero temperature. Right: curves corresponding to the appearance of instability in axial current on the plane $|\boldsymbol{a}|, T$. The area below these curves, presumably, is the forbidden region of the values of acceleration and temperature

Such suppression of the axial current at low temperatures in the region of $\Omega<2(m-|\mu|)$ may be of interest from the experimental point of view.

\section{Acceleration as imaginary chemical potential}

It is known that in theories with an imaginary chemical potential, the periodicity of the partition function as a function of the imaginary chemical potential occurs [19]. It can be expected that a similar periodicity will occur for average values of the observed quantities, as functions 
of acceleration. Indeed, the axial current in (7) turns out to be periodic with $\frac{|a|}{2} \rightarrow \frac{|a|}{2}+2 \pi T n$, $n=0, \pm 1, \pm 2 \ldots$

On the other hand, in [10] it was shown that the Unruh temperature is the minimum possible temperature for an accelerated medium of scalar particles (similar prediction, with twice Unruh temperature, obtained in [11] for fermions). The question arises, what will happen for the fermion medium, and how does the addition of rotation affect this effect? To answer these questions, it is necessary to analyse the formula (9). Due to the terms with integer part, cusps and discontinuities appear in axial current at $T<\widetilde{T}_{U}$. More precisely, periodic instabilities occur for $\frac{g_{a}}{2}=(2 n+1) \pi T, n=0,1,2 \ldots$

The expression (10) sets the boundary temperature for a medium of fermions with rotation and acceleration, below which instabilities appear. In this sense, (10) can be viewed as a generalization of the result $[10,11]$. The boundary temperature for different angles between acceleration and angular velocity $\theta$ is shown on Fig. 1, right. For two cases: $\boldsymbol{\Omega} \| \boldsymbol{a}$ and $\boldsymbol{a}=0$, we get that $\widetilde{T}_{U} \rightarrow T_{U}=\frac{|a|}{2 \pi}$, that is the usual Unruh temperature.

The occurrence of periodic instabilities in (9) corresponds to the theories with an imaginary chemical potential, in which these periodic instabilities are called Roberge-Weiss phase transitions. On the other hand, apparently, these instabilities are associated with UnruhHawking radiation.

An important question is whether the results survive with account of interaction. A full answer would require efforts which go beyond the scope of the present paper. However some comments can be made. When applied to a spinor field in an irreducible representations the boost operator results in a complex number, see, e.g., [20]. Indeed, the angular momentum $\hat{J}$ and boost generator $\hat{K}$ are combined into

$$
\hat{N}=\hat{J}+i \hat{K}, \quad \hat{N}^{\dagger}=\hat{J}-i \hat{K}
$$

where the corresponding eigenvalues $N \neq 0, N^{\dagger}=0$ for left-handed spinors and $N^{\dagger} \neq 0, N=$ 0 for right-handed spinors. This leads to opposite signs of the (imaginary) acceleration for left- and right-handed fermions. Thus, emergence of the imaginary acceleration in Eq. (7) is rooted in general rules of constructing Lorentz-invariant Lagrangian of fermionic fields and, apparently, is not specific for the approximation of free fields.

\section{Conclusions}

In the framework of the quantum-statistical approach [12], we obtained the exact nonperturbative expression for the axial current in a non-stationary medium with thermal vorticity. In the linear approximation, this formula reproduces the well-known expression for CVE. Higher-order corrections were also obtained, with the third-order term in angular velocity corresponding to the expression in the literature.

It is shown that the angular velocity plays the role of an actual chemical potential. Due to this, there is an effect of suppression of axial current in the two-dimensional region $\Omega<$ $2(m-|\mu|)$ at low temperatures.

On the other hand, acceleration appears in the form of imaginary chemical potential. This leads to the appearance of a boundary temperature $\widetilde{T}_{U}$, below which instabilities arise in the axial current. For cases $\Omega \| \boldsymbol{a}$ and $\boldsymbol{a}=0$, these instabilities arise at temperatures below the Unruh temperature, which apparently indicates the occurrence of Unruh-Hawking radiation.

Thus, the CVE is a direct consequence of the distribution functions (3) used in the calculation of the polarization in [12-14]. And since, on the other hand, just CVE underlies the approach to calculating the polarization used in [15], this can be a source of consistency between these two approaches. 
Acknowledgments. Useful discussions with F. Becattini, W. Florkowski, E. Grossi, A. Sorin, E. Speranza and A. Starobinsky are gratefully acknowledged. The work was supported by Russian Science Foundation Grant No 18-02-01107.

\section{References}

[1] A. Vilenkin, Phys. Rev. D 20, 1807 (1979)

[2] D. E. Kharzeev, K. Landsteiner, A. Schmitt and H. U. Yee, Lect. Notes Phys. 871, 1 (2013). arXiv:1211.6245 [hep-ph]

[3] D. T. Son and P. Surowka, Phys. Rev. Lett. 103, 191601 (2009). arXiv:0906.5044 [hep-th]

[4] A. V. Sadofyev, V. I. Shevchenko and V. I. Zakharov, Phys. Rev. D 83, 105025 (2011). arXiv:1012.1958 [hep-th]

[5] V. I. Zakharov, Lect. Notes Phys. 871, 295 (2013). arXiv:1210.2186 [hep-ph]

[6] M. Buzzegoli, E. Grossi and F. Becattini, JHEP 1710, 091 (2017). arXiv:1704.02808 [hep-th]

[7] J. h. Gao, J. y. Pang and Q. Wang. arXiv:1810.02028 [nucl-th]

[8] K. Landsteiner, E. Megias and F. Pena-Benitez, Lect. Notes Phys. 871, 433 (2013). arXiv:1207.5808 [hep-th]

[9] M. Stone and J. Kim, Phys. Rev. D 98(2), 025012 (2018). arXiv:1804.08668 [condmat.mes-hall]

[10] F. Becattini, Phys. Rev. D 97(8), 085013 (2018). arXiv:1712.08031 [gr-qc]

[11] W. Florkowski, E. Speranza and F. Becattini, Acta Phys. Polon. B 49, 1409 (2018). arXiv: 1803.11098 [nucl-th]

[12] F. Becattini, V. Chandra, L. Del Zanna and E. Grossi, Annals Phys. 338, 32 (2013). arXiv:1303.3431 [nucl-th]

[13] W. Florkowski, B. Friman, A. Jaiswal, R. Ryblewski and E. Speranza, Acta Phys. Polon. Supp. 11, 507 (2018). arXiv:1810.01709 [nucl-th]

[14] F. Becattini, I. Karpenko, M. Lisa, I. Upsal and S. Voloshin, Phys. Rev. C 95(5), 054902 (2017). arXiv:1610.02506 [nucl-th]

[15] M. Baznat, K. Gudima, A. Sorin and O. Teryaev, Phys. Rev. C 88(6), 061901 (2013). arXiv:1301.7003 [nucl-th]; 93(3), 031902 (2016). arXiv:1507.04652 [nucl-th]; EPJ Web Conf. 138, 01008 (2017). arXiv:1701.00923

[16] G. Prokhorov and O. Teryaev, Phys. Rev. D 97(7), 076013 (2018). arXiv:1707.02491 [hep-th]

[17] G. Prokhorov, O. Teryaev and V. Zakharov, Phys. Rev. D 98(7), 071901 (2018). arXiv:1805.12029 [hep-th]

[18] W. Florkowski, A. Kumar and R. Ryblewski, Phys. Rev. C 98(4), 044906 (2018). arXiv:1806.02616 [hep-ph]

[19] A. Roberge and N. Weiss, Nucl. Phys. B 275, 734 (1986)

[20] P. Ramond, Front. Phys. 51, 1 (1981) [Front. Phys. 74, 1 (1989)] 